\title{
Gene therapy progress and prospects cancer: oncolytic viruses
}

\author{
T-C Liu ${ }^{1}$ and D Kirn ${ }^{1,2}$ \\ ${ }^{1}$ Department of Clinical Research, Jennerex Biotherapeutics Inc., San Francisco, CA, USA and ${ }^{2}$ Department of Pharmacology, School of \\ Medicine, University of Oxford, Oxford, UK
}

The past 2 years have seen several major advances in oncolytic virotherapy. Studies on the interaction between viruses, immune responses and tumor microenvironment have provided important insight, while clinical trials have shown promise. This review summarizes key findings in this field over the past 2 years, and provides directions for future success.

Gene Therapy (2008) 15, 877-884; doi:10.1038/gt.2008.72; published online 17 April 2008

Keywords: cancer; oncolytic; virus; replication

\section{In brief}

\section{Progress}

- Suppression of innate immune response enhances efficacy

- Carrier cell strategy avoids immune attack

- Targeting tumor microenvironment enhances viral spread and efficacy

- Oncolytic viruses kill cancer stem cells

- Genetic engineering of oncolytic viruses complements chemo-and molecular-targeted therapies

- Genetic engineering of oncolytic viruses targets cancer signaling pathways

- Novel oncolytic virus species are being explored

- A large number of clinical trials have been carried out
Prospects

- Logical design of the next generation of oncolytic viruses may take this strategy to the next level

- Ex vivo studies may predict responses

- The demand for target validation is increasing

- New imaging endpoints in clinical trials.

\section{Introduction}

The use of live viruses for the treatment of cancer dates back to a century. ${ }^{1}$ Cancer-selective oncolytic viruses replicate preferentially in cancer cells and as a result, destroy those cells at the end of replication cycles; normal cells are spared and hence toxicity is limited. Of note, oncolytic viruses can kill apoptosis-resistant tumor cells, and hence do not have cross-resistance with existing therapies. Engineered oncolytic viruses have been developed over the past 15 years and have various mechanisms-of-actions (MOA; Table 1): (1) inherently tumor-selective virus species (for example, RNA viruses, poxviruses); (2) viral gene-deleted mutants-critical viral gene expendable for growth in tumor cells, but not in

Correspondence: Dr D Kirn, Jennerex Biotherapeutics Inc., One Market Street, Spear Tower, Suite 2260, San Francisco, CA 94105, USA.

E-mail: dkirn@jennerex.com

Received 14 January 2008; revised 7 March 2008; accepted 10 March 2008; published online 17 April 2008 normal cells, were deleted (for example, adenovirus dl1520/Onyx-015, herpes simplex virus (HSV) G207); (3) promoter engineered mutants-viral replication was engineered to be dependent on inserted tumor-specific promoters, and as a result, the replication of the virus is restricted to tumor cells that are able to activate the promoters (for example, prostate specific antigen-regulated adenovirus CG7870, telomerase-regulated adenoviruses and HSVs); (4) pseudotyped viruses-normal viral tropism is ablated, and viruses are engineered to attach/bind to specific surface receptors that are expressed exclusively/preferentially on tumor cells (for example, adenovirus Delta-24RGD).

Over the past decade, several oncolytic viruses have been tested in humans, and although the safety results are encouraging, efficacy as single agents was limited. ${ }^{1}$ Possible hurdles include attenuation of the virus caused by genetic engineering of the virus that renders cancer selectivity, host immune responses and lack of understanding of tumor microenvironment. However, H101, an oncolytic adenovirus similar to Onyx-015 (E1B-55K/ 
Table 1 Cancer-selectivity mechanisms of oncolytic viruses

\begin{tabular}{|c|c|c|}
\hline Approach to selectivity & $\begin{array}{l}\text { Agent(s) example and genetic } \\
\text { alterations within virus }\end{array}$ & Genetic target(s) in tumors \\
\hline \multirow{3}{*}{$\begin{array}{l}\text { Inherently tumor-selective } \\
\text { species }\end{array}$} & NDV (none) & IFN resistance \\
\hline & Reovirus (none) & Ras pathway \\
\hline & VSV (none) & IFN resistance \\
\hline \multirow{4}{*}{$\begin{array}{l}\text { Deletion of viral gene that is } \\
\text { necessary for replication in } \\
\text { normal cells, but expendable in } \\
\text { tumor cells }\end{array}$} & G207 (ICP6-/ $\gamma 34.5$-deleted HSV-1) & Proliferation, loss of neurovirulence \\
\hline & Onyx-015 (E1B-55K-/E3B-deleted Ad) & Loss of p53 pathway, late mRNA transport \\
\hline & Delta-24 (E1A-CR2-deleted Ad) & $\begin{array}{l}\text { Loss of } G_{1}-S \text { checkpoint control; loss of pRB } \\
\text { function }\end{array}$ \\
\hline & JX-594 (TK-deleted VV) & Proliferation \\
\hline \multirow{2}{*}{$\begin{array}{l}\text { Tumor-/tissue-specific promoter } \\
\text { engineering to limit viral gene } \\
\text { expression }\end{array}$} & $\begin{array}{l}\text { CG7870 (E1A under rat probasin promoter, } \\
\text { E1B under PSA promoter/enhancer Ad) }\end{array}$ & Prostate cancer \\
\hline & $\begin{array}{l}\text { bM24-TE (Wnt/ } \beta \text {-catenin-promoter/ } \\
\text { enhancer-driven HSV-1) }\end{array}$ & $\begin{array}{l}\text { Wnt/ } \beta \text {-catenin-overexpressing tumors (colorectal, } \\
\text { hepatoblastoma and so on) }\end{array}$ \\
\hline Pseudotyped viruses & $\begin{array}{l}\text { CAR/integrin-binding deleted Ad, replaced with } \\
\text { tumor-targeting ligand }\end{array}$ & Tumor-specific receptor \\
\hline
\end{tabular}

Abbreviations: CAR, coxsackievirus-adenovirus receptor; IFN, interferon; NDV, Newcastle disease virus; VSV, vesicular stomatitis virus.

E3B-deleted), was recently approved by the Chinese government to be used in conjunction with radiation therapy for the treatment of head and neck cancers. This is the first oncolytic virus product approved by a governmental agency for human use. To overcome the obstacles toward efficacious virotherapeutics, several major advances have been made to improve the selectivity and efficacy of oncolytic viruses. This review summarizes recent major advances over the past 2 years from over 400 publications and selected unpublished work.

\section{Progress}

\section{Suppression of innate immune response enhances efficacy}

Virus-immune system interactions have been extensively studied in the context of virotherapy. Innate immune responses to the virus are a major hurdle for long-term gene expression and oncolytic potency. The use of immunomodulatory agents in combination with oncolytic viruses was first reported in the 1970s. Recently, several groups have shown, using different viruses and animal models, that administration of cyclophosphamide, known to inhibit innate immune responses, can significantly enhance viral spread, transgene expression and antitumoral efficacy. These studies demonstrated that cyclophosphamide is able to inhibit neutralizing antibody induction, macrophages, regulatory T cells (Tregs) induction and intratumoral interferon (IFN) $-\gamma$ production..$^{2-8}$ It remains to be determined, however, how much this approach will benefit cancer patients who often have already various degrees of 'pre-existing' immunosuppression due to disease and chemotherapy.
In addition, Haralambieva et al. ${ }^{9}$ showed that measles virus-induced gene expression and intratumoral virus spread is inhibited by IFN, which is triggered by virus infection of tumor cells. Interestingly, currently available oncolytic measles viruses are derived from Edmonston tag (Edmtag) strain, which has lost most of the IFNantagonizing activities. Edmtag-based measles virus engineered to express the measles phosphoprotein $(\mathrm{P})$ gene products $(\mathrm{P} / \mathrm{V} / \mathrm{C}$ proteins) from wild-type measles virus, known to antagonize IFN induction and response, exhibited reduced IFN sensitivity and a reduced IFN induction in lymphoma, myeloma and activated peripheral blood mononuclear cells (PBMC). ${ }^{9}$ Measles virus encoding the $\mathrm{P}$ gene products also showed significantly enhanced systemic efficacy in a myeloma xenograft model. This study highlights the importance of innate antiviral responses of tumor cells that need to be considered when designing oncolytic viruses.

Importantly, antiviral immunity does not necessarily reduce the efficacy of virus. A recent report by Zhu et al. ${ }^{10}$ demonstrated that in mice pre-immunized with HSV, subsequent intratumoral administration of oncolytic HSV showed enhanced efficacy compared to HSVnaive mice. The peripheral blood mononuclear cells from the HSV-seropositive mice also exhibited greater in vitro cytotoxicity to tumor cells than PBMC from HSV-naive mice, which correlated with an enhanced IFN- $\gamma$ induction in PBMC from HSV-seropositive mice. ${ }^{10}$ This is an important finding that needs to be explored with systemic HSV administration and also with other oncolytic virus species.

Consistent with our previous findings, Diaz et al. ${ }^{11}$ have shown that host CD8 and NK cells are critical for the efficacy of oncolytic virotherapy. Importantly, using an antibody against Treg (PC-61) they showed that depletion of Treg cells inhibited antitumoral efficacy in 
the context of virotherapy, as it overcame the suppression of antiviral immune responses. Furthermore, levels of activated T cells can be significantly increased by enhanced expression of tumor antigens. This can be achieved either through adoptive T-cell transfer therapy, or incorporation of tumor antigen into the oncolytic virus. ${ }^{11}$

\section{Carrier cell strategy avoids immune attack}

In addition to blocking the host immune response, one can take advantage of the immune system to boost antitumor responses. Adenovirus and HSV mutants engineered to enhance the expression of class I major histocompatibility complex (MHC) have been shown to enhance enhanced antitumoral immune responses and efficacy in animal models. However, one major challenge for virotherapy is the inefficient uptake of viruses into tumor cells after systemic administration due to systemic antiviral immune response (for example, neutralizing antibodies and complement). Thorne et al. ${ }^{12}$ described a novel approach to tackle this issue. Cytokine-induced killer (CIK) cells are known to 'home' to and destroy tumors. After isolating the CIK cells from mice, these cells were infected with oncolytic vaccinia viruses and readministered into tumor-bearing animals. The virus replicated in the CIK cells while these traveled to the tumors. As a result, substantially larger amounts of oncolytic viruses were delivered to the tumor, and both the CIK cells and oncolytic viruses were synergistic in tumor killing. ${ }^{12}$ It remains to be seen, however, whether CIK cells home to tumors in humans within a reasonable time frame. In addition, this approach requires harvesting of cells from individual patients, ex vivo culturing and redelivery to the patients, and therefore requires a substantial amount of laboratory work. Nonetheless, this strategy holds promise.

Subsequently, the 'carrier cell' approach has been tested by several groups. ${ }^{13}$ Using measles viruses, Ong et al. ${ }^{14}$ showed that virus-infected T cells can protect from low, but not high, concentrations of antimeasles immune serum. However, even in measles-naive mice, only $1-2 \%$ of the virus-infected $\mathrm{T}$ cells trafficked to the tumor site after systemic delivery. Power et $a l .{ }^{15}$ tested oncolytic vesicular stomatitis virus (VSV) vectors with different carrier cell types, ranging from leukemia cell lines (which led to systemic delivery of the virus) to cells derived from solid tumors (which accumulated primarily in the lungs). Using dual-enzyme in vivo luminescence imaging, it was shown that whereas the carrier cells were retained in the body for no longer than 1 day, oncolytic VSV continued to replicate and was able to eradicate pre-established tumors. ${ }^{15}$ Other carrier cells tested include endothelial cells and mesenchymal stem cells. ${ }^{16,17}$ Vile et al. have recently shown that T lymphocytes can be used to harbor oncolytic VSV and release the virus at the tumor site. ${ }^{18}$ VSV-loaded lymphocytes were also able to purge spleen and lymph nodes of metastatic cells, which in turn primed antitumoral immunity. Furthermore, adoptive transfer of VSV-loaded lymphocytes reduced metastases. ${ }^{18}$ This strategy might have great potential for many tumor types.

While the 'Trojan Horse' approach avoids the negative impacts of neutralizing antibodies on virotherapy, there are several critical issues that need to be further investigated before this approach can be taken into humans. First of all, the most appropriate carrier cell types need to be carefully determined. Tumor-homing cells (for example, CIK) and systemic disseminating cells (for example, leukemic cells) have both advantages and disadvantages. Importantly, tumorigenicity is a major concern when cancer cells are used as carriers. Finally, although viruses are able to replicate, the newly generated viruses (that is, 'second wave') still face contact with neutralizing antibodies, which will likely restrict significant virus spread. Multiple administrations of viruses in carrier cells may, therefore, be necessary. Finally, whether or not viral antigens will be presented by carrier cells, and the impact on virus delivery and replication, needs to be explored.

\section{Targeting the tumor microenvironment enhances viral spread and efficacy}

The efficacy of virotherapy can be limiting when replication-mediated oncolysis is the sole MOA. Indeed, the tumor microenvironment plays an important role in restricting viral spread and promoting tumor growth. To address this issue, several approaches have been taken. The first is to engineer viral vectors with therapeutic transgenes that target the key components of the tumor microenvironment (for example, the tumor vasculature or matrix). Oncolytic adenovirus encoding relaxin, a matrix-degrading protein, was able to enhance viral spread without causing significant toxicity. ${ }^{19}$ Oncolytic HSV encoding dominant-negative fibroblast growth factor receptor or antiangiogenic protein platelet factor-4 led to significant reduction in tumor vasculature and as a result, significantly enhanced therapeutic efficacy. ${ }^{20,21}$ Others have engineered oncolytic virus replication to be activated by tumor matrix metalloproteinases (MMP), ${ }^{22}$ and shown that MMP-8 gene delivery enhanced the efficacy of oncolytic adenovirus. ${ }^{23}$

An alternative approach is to coadminister therapeutic agents with the virus. Coadministration of matrixmodifying agents (bacterial collagenase, MMP-1, 8) has been shown to enhance the spread of oncolytic HSV, 24,25 although concerns about tumor metastases have to be explored in more preclinical models before translation into clinical trials. In addition, infection with wild-type HSV results in reduction in thrombospondin secretion, a protein that has antiangiogenic properties, from extracellular matrix. This leads to increased vascularity in infected tissues. Aghi et al. ${ }^{26}$ showed that increased vascularity can be counteracted by introduction of certain mutations into oncolytic HSV, or coadministering a thrombospondin-derived peptide 3TSR. A recent study by Kolodkin-Gal et al. ${ }^{27}$ showed that the difference in amount of extracellular matrix between normal colon and colon cancer tissues determined the infectivity and subsequent cytotoxicity of HSV. This phenomenon needs to be further investigated.

Tumor hypoxia and its impact on viral replication have also been studied. Previous reports have shown that hypoxia limits group $\mathrm{C}$ adenovirus (serotype 5) replication, and new data suggest that the oncolytic activity of other adenovirus serotypes are also affected. ${ }^{28}$ Importantly, the report showed that the expression level of CD46, a receptor for group B adenoviruses (serotypes 3,11 ) as well as measles virus, was not altered in hypoxic conditions. In contrast, we have found that hypoxia 
enhances the replication of oncolytic HSV (M Aghi et al., unpublished).

Another important issue is to explore how inflammation induced by virus infection impacts on the tumor microenvironment. Breitbach et al. ${ }^{29}$ showed that administration with VSV and vaccinia viruses resulted in a dramatic transcriptional activation of the proinflammatory neutrophil chemoattractants CXCL1 and CXCL5 and neutrophil attraction. The neutrophils in turn contributed to acute reduction in tumor vasculature. Targeted recruitment of neutrophils to infected tumor beds enhances the killing of malignant cells. ${ }^{29}$ Recent work by Kurozumi et al. ${ }^{30}$ also illustrates the importance of targeting the tumor microenvironment to improve the efficacy of oncolytic virotherapy. Using the orthotopic (that is, tumors grown in the organs where it is derived from) immunocompetent rat glioma model, they showed that oncolytic HSV infection increased tumor vascular permeability, host leukocyte infiltration into tumors and intratumoral expression of inflammatory cytokine genes, all of these were part of the inflammatory response after HSV infection. Pretreatment with cyclophosphamide suppressed the inflammation and resulted in reduced tumor vascular permeability. ${ }^{30}$

Kirn et al. ${ }^{31}$ showed that systemically administered vaccinia virus resulted in infection and subsequent destruction of tumor endothelial cells, which led to loss of tumor vascular density.

\section{Oncolytic viruses kill cancer stem cells}

In light of recent discoveries in the filed of cancer stem cells, it is becoming clear that those cell populations not only initiate tumorigenesis, but also contribute importantly to resistance to chemo- and radiation therapy. As this cell population is capable of replication and selfrenewal, oncolytic viruses that are designed to target cell cycle-dysregulated tumor cells might also possess the ability to kill cancer stem cells. Indeed, several recent publications have shown that the adenovirus E1A mutant that targets the retinoblastoma-E2F transcriptional factor pathway (Delta-24) is able to kill CD133+ cancer stem cells or CD44(+)/CD22(-/low) cancer initiating cells in vitro, and is also able to eradicate tumors derived from these cancer stem cells. ${ }^{32,33} \mathrm{MOA}$ include replication-induced cell lysis (necrosis) and autophagy (degradation of intracellular components in lysosomes). ${ }^{32}$ It has also been reported that adenovirus serotype 3 was better than serotype 5 in infecting cancer stem cells in vitro. This ability to kill cancer stem cells does not seem to be limited to adenoviruses, as oncolytic HSV can also efficiently kill glioma stem cells (H Wakimoto et al., unpublished). While this is of interest, there are several issues that remain to be solved. As the population of cancer stem cells within a tumor is generally low (often less than 5\%), it is a challenge for oncolytic viruses to 'find' and kill these cancer stem cells, especially when viruses are administered systemically. Secondly, there is so far no direct evidence of anticancer stem cells efficacy of this approach in vivo in tumors.

\section{Genetic engineering of oncolytic viruses complements chemo- and molecular-targeted therapies}

Several novel combination treatments have been tested in combination with oncolytic viruses. Genetic engineering of the viruses allows functional complementation to chemotherapeutic agents and molecular-targeted therapeutics. Aghi et al. ${ }^{34}$ showed that temozolomide-induced DNA repair pathways in glioma cells complemented replication of $\gamma 34.5$-deleted oncolytic HSV replication and resulted in enhanced efficacy both in vitro and in vivo. Stanford et al. ${ }^{35}$ and Lun et al. ${ }^{36}$ showed that treatment of rapamycin, a mammalian target of rapamycin inhibitor which resulted in increased Akt/protein kinase $B$ activation, one was able to enhance myxoma virus replication in tumor, but not normal, cells. We showed that by deleting Us3, oncolytic HSV can synergize with Phosphoinositide-3 kinase (PI3K)/Akt inhibitors in vitro and have enhanced efficacy without increasing toxicity in vivo. ${ }^{37}$

Histone deacetylase (HDAC) inhibitors are currently being investigated in combination with various oncolytic virus species. Trichostatin A (TSA) has been shown to upregulate cellular coxsackievirus-adenovirus receptor (CAR) expression and hence, infectibility of tumor cells to adenoviruses. We showed that in addition to CAR upregulation, TSA possesses antitumoral and antiangiogenic activities, and shows synergistic tumor-killing effect with oncolytic HSVs in vitro and enhanced efficacy in vivo (Liu et al. ${ }^{38}$ ). In contrast, valproic acid, another HDAC inhibitor used for epilepsy disorders, showed antagonistic effect with oncolytic adenoviruses in vitro, presumably due to enhanced apoptosis that limits viral replication. ${ }^{39}$ It will be interesting to see what the effect other types of HDAC inhibitors will have on different oncolytic virus species.

\section{Genetic engineering of oncolytic viruses targets cancer signaling pathways}

Through genetic engineering, viruses can be designed to target cancer cells through certain activated signaling pathways. Apart from the Akt pathway targeted by myxoma virus described above, HSV $\gamma 34.5$-deleted mutants showed enhanced replication in cells with activated mitogen-activated protein (MAP) kinase or extracellular signal-regulated (ERK) kinase, which in turn inhibited protein kinase $\mathrm{R}$ activity, thus circumventing the negative impact of the IFN signaling pathway. ${ }^{40,41}$ Similarly, viruses such as VSV showed preferential replication in cells with an activated Ras-ERK pathway and defective IFN pathways. ${ }^{42}$ A vaccinia virus mutant with a deletion in B18R, whose gene product neutralizes type I IFNs, showed IFN-dependent cancer selectivity and efficacy. ${ }^{31}$ It has also been shown that adenovirusinduced ERK activation is critical to viral replication. ${ }^{43}$ Oncolytic viruses can also be 'programmed' to replicate in cells through certain cellular signaling activities, such as $\beta$-catenin, ${ }^{44}$ to carry therapeutic transgene that targets tumorigenic pathways, ${ }^{20}$ or retargeted to cellular receptors that are essential for signaling (for example, epidermal growth factor receptor (EGFR)). ${ }^{45}$

\section{Novel oncolytic virus species are being explored}

As most oncolytic viruses have produced less than optimal efficacy in clinical trials as single agents, there is great interest in exploring novel viral species. These studies assess oncolytic activity and/or investigate tumor selectivity. For example, the porcine Seneca Valley virus has recently been discovered to possess anti- 
tumoral activity against certain cancers of neuroendocrine origin. ${ }^{46}$ It has been speculated that the tumor selectivity is based on differential receptor binding in cancer and normal cells, but more work needs to be done to verify this and to study the impact of the immune system has on the virus. Myxoma virus, a rabbit virus, has also been assessed as an oncolytic agent. New studies reveal that the tumor selectivity of myxoma virus is based on overexpression of Akt in human cancer cells, which facilitates replication and oncolysis. ${ }^{47}$ While these are important findings, the safety profiles of these viruses need to be cautiously examined, especially when the natural host of the virus is not human.

Intratumoral administration of UV-inactivated, replication-deficient Sendai virus induced a robust antitumoral immune response (including cytotoxic T lymphocyte (CTL) induction, dendritic cell maturation and antagonism of Tregs) and resulted in significant efficacy in CT26 syngeneic murine colorectal cancer model. ${ }^{48}$ It will be interesting to study how much of this vaccine effect contributes to antitumoral efficacy in oncolytic Sendai virus studies. A 'vaccine' effect was also seen with parvovirus $\mathrm{H}-1$ in a rat lung tumor metastases model. ${ }^{49}$

\section{A large number of clinical trials have been carried out} Virotherapy has several features that are distinct from other therapeutics. Its multiple novel MOAs include replication-mediated oncolysis, antitumoral immunity induction, antiangiogenesis, apoptosis and autophage induction. There is no cross resistance with other therapeutics, and synergistic interaction is seen with other treatment modalities. Safety in human has been demonstrated in more than 800 patients. In addition, current biotechnology allows us to rapidly address issues encountered in clinics at the bench. There are several reports on virotherapy clinical trials over the past 2 years. Readers are referred to other articles for a more comprehensive review. ${ }^{1}$ A list of oncolytic virus agents that have completed, or are currently in late phase trials, are listed in Table 2 . In a recent study examining the OV 001 (HUJ) strain of Newcastle disease virus (NDV), OV 001 was administered intravenously to 11 patients with glioblastoma with no dose-limiting toxicity. ${ }^{50}$ Following biweekly maintenance therapy, one complete remission with a duration of 3 months was described. Virus was recovered from blood, urine and saliva samples. Infectious NDV was recovered from a tumor biopsy. In a separate study testing intravenous delivery of a different NDV strain PV701, using 'two-step' desensitization (dosing with significantly smaller doses prior to full dose) has proven to significantly reduce acute adverse events. $^{51}$

The HSV-1 mutant NV1020 (R7020) virus was originally developed as a vaccine. The virus has a deletion in one of the two copies of the $\gamma-34.5$ gene. A phase I trial of NV1020 administered by hepatic arterial infusion (HAI) was performed in HSV-seropositive patients with colorectal liver metastases. ${ }^{52}$ No significant toxicity was noted in patients receiving doses up to $1 \times 10^{8}$ pfu per infusion. No replication data were reported. An ongoing phase I/II trial is evaluating repeat HAI of NV1020 followed by second-line chemotherapy in seropositive patients with colorectal cancer. The oncolytic HSV vector OncoVEXGM-CSF has been generated with deletions in $\gamma-34.5$ (to reduce pathogenicity) and ICP47 (to restore MHC I presentation). In addition, OncoVEX $X^{\mathrm{GM}-\mathrm{CSF}}$ has a granulocyte monocyte colony-stimulating factor (GMCSF) transgene insertion. An early passage clinical isolate was used to generate OncoVEX ${ }^{\mathrm{GM}-\mathrm{CSF}}$ because it had enhanced potency relative to available laboratory strains. A phase I trial of intratumoral injection of OncoVEX ${ }^{\mathrm{GM}-\mathrm{CSF}}$ into cutaneous metastases from solid tumors and melanomas was carried out. ${ }^{53}$ Treatment did not result in significant systemic toxicities; injection-site inflammation was dose limiting. Viral genomes were detected shedding from the skin over ulcerated tumors. Injected tumor histology showed inflammation and necrosis. No distant efficacy was reported. Phase II trials of intratumoral injection using OncoVEX ${ }^{\mathrm{GM}-\mathrm{CSF}}$ are underway in patients with melanoma and other tumor types. A Phase III trial of HSV-1 mutant 1716 in brain tumor has also been announced.

In addition, an oncolytic vaccinia virus with deletion in thymidine kinase (TK) and expressing GM-CSF, JX-594, has been tested in patients with liver tumors. Unlike other virus species described above, systemic exposure and evidence of replication in vivo has been

Table 2 Oncolytic viral agents in completed or ongoing late phase trials

\begin{tabular}{|c|c|c|c|c|}
\hline Product & Species & Genetic modification & Target tumor type & Phase \\
\hline Reolysin & Reovirus & None & Bone/soft-tissue sarcoma & II \\
\hline NDV (MTH-68H) & Newcastle disease virus & None & Metastatic solid tumors & II \\
\hline JX-594 & Vaccinia & Thymidine kinase deletion; & 1. Hepatocellular & II \\
\hline & & GM-CSF expression & 2. Melanoma & II \\
\hline H101 & Adenovirus & E1B-55 K, E3 deletion & $\begin{array}{l}\text { Head and neck } \\
\text { (+ chemotherapy) }\end{array}$ & III \\
\hline $\begin{array}{l}\text { Ad5-yCD/ } \\
\text { mutTKSR39rep-ADP }\end{array}$ & Adenovirus & $\begin{array}{l}\text { E1B- } 55 \mathrm{~K} \text { deletion; CD/TK } \\
\text { fusion gene expression; ADP } \\
\text { overexpression }\end{array}$ & Prostate (+ radiotherapy) & II \\
\hline OncoVex ${ }^{\mathrm{GM}-\mathrm{CSF}}$ & HSV 1 & $\begin{array}{l}\gamma 34.5 \text { and ICP47 deletion; } \\
\text { GM-CSF expression }\end{array}$ & Melanoma & II \\
\hline 1716 & HSV 1 & $\gamma 34.5$ deletion & Brain & III \\
\hline
\end{tabular}

Abbreviations: ADP, adenovirus death protein; GM-CSF, granulocyte monocyte colony-stimulating factor; HSV, herpes simplex virus; $\mathrm{TK}$, thymidine kinase; VSV, vesicular stomatitis virus. 
demonstrated in this study. Tumor response was observed in the majority of treated tumors, and distant tumor responses were demonstrated in several patients with target tumor responses. Replication and biological activity in vivo have also been shown. ${ }^{54}$ With the advance in our knowledge of cancer and oncolytic virus biology, we expect that carefully designed clinical studies will not only show proof-of-concept for this novel treatment, but also result in evidence of clinical benefit in patients.

\section{Prospects}

\section{Logical design of the next generation of oncolytic viruses may take this strategy to the next level}

Design of the next generation of oncolytic viruses should be based on current knowledge of virology, immunology and cancer biology. More importantly, findings from clinical trials should be incorporated/addressed. Two recent publications illustrate this concept. ${ }^{31,55}$ The first article describes rational species and strain selection and genetic engineering based on updated knowledge. As described before, poxvirus was selected for this study based on human data showing that systemic delivery of poxvirus is safe and can induce significant tumor responses. ${ }^{56} \mathrm{~A}$ highly potent vaccinia virus strain that also trafficked efficiently to human tumors after intravenous administration was first identified. This strain was then engineered to target cancer cells with activated transcription factor E2F and the EGFR pathway, and further engineered to express human GM-CSF for induction of tumor-specific CTL. The new vaccinia construct, JX-963, demonstrated significant cancer selectivity in human tumor cell lines, tumor-bearing rabbits and primary human surgical samples. Intravenous administration led to systemic efficacy against both primary carcinomas and widespread organ-based metastases in immunocompetent animals. ${ }^{55}$ The second study describes the use of a vaccinia virus background that selectively targets IFN pathway resistance in tumor cells. Further engineering with TK deletion and IFN- $\beta$ insertion results in a multimechanistic oncolytic vaccinia virus. $^{31}$

\section{Ex vivo studies may predict responses}

Genetic markers are being developed for chemotherapy and molecular-targeted therapeutics to predict responses and/or idiosyncratic reactions, and the results have been implemented into practice. Given the complexity of MOA of virotherapeutics, there is therefore a long way to go before such markers/predictors can be developed for virotherapy. However, testing patients' samples ex vivo prior to treatment may provide important information and complement our current studies. In addition to the difference between results obtained from immortalized cell lines and primary cancer cells, explant samples contain extracellular matrix and a three-dimensional structure that more closely mimics clinical situation. Importantly, when adjacent normal tissues are included, a therapeutic index can be obtained, which is critical for local administration protocol. Several recent publications described the use of this approach. ${ }^{27,55,57,58}$ The results of oncolytic viruses on ex vivo tissues and its clinical outcome correlation have yet to be established, but researchers are encouraged to include explants studies whenever possible to maximize clinical relevance. Future clinical developments might include pre-/post-treatment ex vivo assessment of infectivity, cytopathic effects and viral replication.

\section{The demand for target validation is increasing}

The demand for target validation in small moleculebased kinase inhibitors is increasing. For virotherapy to be successful, it has to pass similar hurdles. The most important biological endpoint that needs to be demonstrated with all species of oncolytic virus is tumor-selective virus replication, therapeutic transgene expression and biological function (if applicable). This has been effectively achieved with several virus species, but still required for others. Depending on the strategy demonstration of targeting cancer-specific features pathways may also be necessary. For instance, if viruses are designed to target the Ras/Raf/MAPK pathway, a reduction in pathway activity should be proven. High TK activity in cancer cells are needed for TK-deleted viruses, while viruses replicating exclusively in IFNresistant cells (cancer cells) need to demonstrate local IFN induction in vivo. These are difficult tasks but warrant further exploration. The effect of oncolytic viruses on tumor microenvironment, as shown in various preclinical studies, will need to be validated in clinical trials. For instance, future clinical studies should include tumor vascularity assessment to see if virotherapy reduces tumor vasculature.

\section{New imaging endpoints in clinical trials?}

Tumor size measurement has been a gold standard for defining responses in clinical practice. Tumor progression is defined as increased in tumor size above certain degrees. However, recent studies on molecular therapeutics have shown that some agents induce tumor necrosis without affecting the sizes of the tumors. To address this issue, new criteria (for example, Choi criteria $^{59}$ ) have been incorporated in tumor response assessment by imaging. Since most of the virotherapy clinical trials were done by local or locoregional administration, it is likely that the effect of the viruses is localized within the tumors. Thus, we need to consider whether adopting these new criteria is feasible. Correlation between tumor size, tumor density and survival will be necessary in late phase trials.

\section{Abbreviations}

CAR, coxsackievirus-adenovirus receptor; CIK, cytokineinduced killer; CTL, cytotoxic T lymphocyte; EGFR, epidermal growth factor receptor; HDAC, histone deacetylase; HSV, herpes simplex virus; IFN, interferon; MMP, matrix metalloproteinase; MOA, mechanism-ofaction; PBMC, peripheral blood mononuclear cells; TK, thymidine kinase; Treg, regulatory T cells; TSA, trichostatin A; VSV, vesicular stomatitis virus

\section{References}

1 Liu TC, Galanis E, Kirn D. Clinical trial results with oncolytic virotherapy: a century of promise, a decade of progress. Nat Clin Pract Oncol 2007; 4: 101-117. 
2 Fulci G, Breymann L, Gianni D, Kurozomi K, Rhee SS, Yu J et al. Cyclophosphamide enhances glioma virotherapy by inhibiting innate immune responses. Proc Natl Acad Sci USA 2006; 103: 12873-12878

3 Qiao J, Wang H, Kottke T, White C, Twigger K, Diaz RM et al. Cyclophosphamide facilitates antitumor efficacy against subcutaneous tumors following intravenous delivery of reovirus. Clin Cancer Res 2008; 14: 259-269.

4 Lamfers ML, Fulci G, Gianni D, Tang Y, Kurozumi K, Kaur B et al. Cyclophosphamide increases transgene expression mediated by an oncolytic adenovirus in glioma-bearing mice monitored by bioluminescence imaging. Mol Ther 2006; 14: 779-788.

5 Fulci G, Dmitrieva N, Gianni D, Fontana EJ, Pan X, Lu Y et al. Depletion of peripheral macrophages and brain microglia increases brain tumor titers of oncolytic viruses. Cancer Res 2007; 67: 9398-9406.

6 Li H, Zeng Z, Fu X, Zhang X. Coadministration of a herpes simplex virus-2 based oncolytic virus and cyclophosphamide produces a synergistic antitumor effect and enhances tumorspecific immune responses. Cancer Res 2007; 67: 7850-7855.

7 Ungerechts G, Springfeld C, Frenzke ME, Lampe J, Parker WB, Sorscher EJ et al. An immunocompetent murine model for oncolysis with an armed and targeted measles virus. Mol Ther 2007; 15: 1991-1997.

8 Di Paolo NC, Tuve S, Ni S, Hellstrom KE, Hellstrom I, Lieber A. Effect of adenovirus-mediated heat shock protein expression and oncolysis in combination with low-dose cyclophosphamide treatment on antitumor immune responses. Cancer Res 2006; 66: 960-969.

9 Haralambieva I, Iankov I, Hasegawa K, Harvey M, Russell SJ, Peng KW. Engineering oncolytic measles virus to circumvent the intracellular innate immune response. Mol Ther 2007; 15: 588-597.

10 Zhu H, Su Y, Zhou S, Xiao W, Ling W, Hu B et al. Immune analysis on mtHSV mediated tumor therapy in HSV-1 seropositive mice. Cancer Biol Ther 2007; 6: 724-731.

11 Diaz RM, Galivo F, Kottke T, Wongthida P, Qiao J, Thompson J et al. Oncolytic immunovirotherapy for melanoma using vesicular stomatitis virus. Cancer Res 2007; 67: 2840-2848.

12 Thorne SH, Negrin RS, Contag CH. Synergistic antitumor effects of immune cell-viral biotherapy. Science 2006; 311: 1780-1784.

13 Power AT, Bell JC. Cell-based delivery of oncolytic viruses: a new strategic alliance for a biological strike against cancer. Mol Ther 2007; 15: 660-665.

14 Ong HT, Hasegawa K, Dietz AB, Russell SJ, Peng KW. Evaluation of T cells as carriers for systemic measles virotherapy in the presence of antiviral antibodies. Gene Therapy 2007; 14: 324-333.

15 Power AT, Wang J, Falls TJ, Paterson JM, Parato KA, Lichty BD et al. Carrier cell-based delivery of an oncolytic virus circumvents antiviral immunity. Mol Ther 2007; 15: 123-130.

16 Iankov ID, Blechacz B, Liu C, Schmeckpeper JD, Tarara JE, Federspiel MJ et al. Infected cell carriers: a new strategy for systemic delivery of oncolytic measles viruses in cancer virotherapy. Mol Ther 2007; 15: 114-122.

17 Hakkarainen T, Sarkioja M, Lehenkari P, Miettinen S, Ylikomi T, Suuronen $\mathrm{R}$ et al. Human mesenchymal stem cells lack tumor tropism but enhance the antitumor activity of oncolytic adenoviruses in orthotopic lung and breast tumors. Hum Gene Ther 2007; 18: 627-641.

18 Qiao J, Kottke T, Willmon C, Galivo F, Wongthida P, Diaz RM et al. Purging metastases in lymphoid organs using a combination of antigen-nonspecific adoptive $\mathrm{T}$ cell therapy, oncolytic virotherapy and immunotherapy. Nat Med 2008; 14: 37-44.

$19 \mathrm{Kim} \mathrm{JH}$, Lee YS, Kim H, Huang JH, Yoon AR, Yun CO. Relaxin expression from tumor-targeting adenoviruses and its intratumoral spread, apoptosis induction, and efficacy. J Natl Cancer Inst 2006; 98: 1482-1493.
20 Liu TC, Zhang T, Fukuhara H, Kuroda T, Todo T, Canron X et al. Dominant-negative fibroblast growth factor receptor expression enhances antitumoral potency of oncolytic herpes simplex virus in neural tumors. Clin Cancer Res 2006; 12: 6791-6799.

21 Liu TC, Zhang T, Fukuhara H, Kuroda T, Todo T, Martuza RL et al. Oncolytic HSV armed with platelet factor 4, an antiangiogenic agent, shows enhanced efficacy. Mol Ther 2006; 14: 789-797.

22 Springfeld C, von Messling V, Frenzke M, Ungerechts G, Buchholz CJ, Cattaneo R. Oncolytic efficacy and enhanced safety of measles virus activated by tumor-secreted matrix metalloproteinases. Cancer Res 2006; 66: 7694-7700.

23 Cheng J, Sauthoff H, Huang Y, Kutler DI, Bajwa S, Rom WN et al. Human matrix metalloproteinase- 8 gene delivery increases the oncolytic activity of a replicating adenovirus. Mol Ther 2007; 15: 1982-1990.

24 McKee TD, Grandi P, Mok W, Alexandrakis G, Insin N, Zimmer JP et al. Degradation of fibrillar collagen in a human melanoma xenograft improves the efficacy of an oncolytic herpes simplex virus vector. Cancer Res 2006; 66: 2509-2513.

25 Mok W, Boucher Y, Jain RK. Matrix metalloproteinases-1 and -8 improve the distribution and efficacy of an oncolytic virus. Cancer Res 2007; 67: 10664-10668.

26 Aghi M, Rabkin SD, Martuza RL. Angiogenic response caused by oncolytic herpes simplex virus-induced reduced thrombospondin expression can be prevented by specific viral mutations or by administering a thrombospondin-derived peptide. Cancer Res 2007; 67: 440-444.

27 Kolodkin-Gal D, Zamir G, Pikarski E, Pikarski A, Shimony N, $\mathrm{Wu} \mathrm{H}$ et al. A novel system to study adenovirus tropism to normal and malignant colon tissues. Virology 2007; 357: 91-101.

28 Shen BH, Bauzon M, Hermiston TW. The effect of hypoxia on the uptake, replication and lytic potential of group B adenovirus type 3 (Ad3) and type 11p (Ad11p). Gene Therapy 2006; 13: 986-990.

29 Breitbach CJ, Paterson JM, Lemay CG, Falls TJ, McGuire A, Parato KA et al. Targeted inflammation during oncolytic virus therapy severely compromises tumor blood flow. Mol Ther 2007; 15: $1686-1693$.

30 Kurozumi K, Hardcastle J, Thakur R, Yang M, Christoforidis G, Fulci $\mathrm{G}$ et al. Effect of tumor microenvironment modulation on the efficacy of oncolytic virus therapy. J Natl Cancer Inst 2007; 99: 1768-1781.

31 Kirn DH, Wang Y, Le Boeuf F, Bell J, Thorne SH. Targeting of interferon-beta to produce a specific, multi-mechanistic oncolytic vaccinia virus. PLoS Med 2007; 4: e353.

32 Jiang H, Gomez-Manzano C, Aoki H, Alonso MM, Kondo S, McCormick F et al. Examination of the therapeutic potential of Delta-24-RGD in brain tumor stem cells: role of autophagic cell death. J Natl Cancer Inst 2007; 99: 1410-1414.

33 Eriksson $M$, Guse $K$, Bauerschmitz G, Virkkunen $P$, Tarkkanen $\mathrm{M}$, Tanner $\mathrm{M}$ et al. Oncolytic adenoviruses kill breast cancer initiating CD44(+)CD24(-/low) cells. Mol Ther 2007; 15: 2088-2093.

34 Aghi M, Rabkin S, Martuza RL. Effect of chemotherapy-induced DNA repair on oncolytic herpes simplex viral replication. J Natl Cancer Inst 2006; 98: 38-50.

35 Stanford MM, Barrett JW, Nazarian SH, Werden S, McFadden G. Oncolytic virotherapy synergism with signaling inhibitors: rapamycin increases myxoma virus tropism for human tumor cells. J Virol 2007; 81: 1251-1260.

36 Lun XQ, Zhou H, Alain T, Sun B, Wang L, Barrett JW et al. Targeting human medulloblastoma: oncolytic virotherapy with myxoma virus is enhanced by rapamycin. Cancer Res 2007; 67: 8818-8827.

37 Liu TC, Wakimoto H, Martuza RL, Rabkin SD. Herpes simplex virus us3(-) mutant as oncolytic strategy and synergizes with phosphatidylinositol 3-kinase-akt targeting molecular therapeutics. Clin Cancer Res 2007; 13: 5897-5902. 
38 Liu TC, Castelo-Branco P, Rabkin SD, Martuza RL. Trichostatin A and oncolytic HSV combination therapy shows enhanced antitumoral and antiangiogenic effects. Mol Ther 2008; e-pub ahead of print 1 April 2008; doi:10.1038/mt.2008.33.

39 Hoti N, Chowdhury W, Hsieh JT, Sachs MD, Lupold SE, Rodriguez R. Valproic acid, a histone deacetylase inhibitor, is an antagonist for oncolytic adenoviral gene therapy. Mol Ther 2006; 14: 768-778.

40 Smith KD, Mezhir JJ, Bickenbach K, Veerapong J, Charron J, Posner MC et al. Activated MEK suppresses activation of PKR and enables efficient replication and in vivo oncolysis by Deltagamma(1)34.5 mutants of herpes simplex virus 1. J Virol 2006; 80: 1110-1120.

41 Veerapong J, Bickenbach KA, Shao MY, Smith KD, Posner MC, Roizman B et al. Systemic delivery of (gamma1)34.5-deleted herpes simplex virus-1 selectively targets and treats distant human xenograft tumors that express high MEK activity. Cancer Res 2007; 67: 8301-8306.

42 Noser JA, Mael AA, Sakuma R, Ohmine S, Marcato P, Lee PW et al. The RAS/Raf1/MEK/ERK signaling pathway facilitates VSV-mediated oncolysis: implication for the defective interferon response in cancer cells. Mol Ther 2007; 15: 1531-1536.

43 Schumann M, Dobbelstein M. Adenovirus-induced extracellular signal-regulated kinase phosphorylation during the late phase of infection enhances viral protein levels and virus progeny. Cancer Res 2006; 66: 1282-1288.

44 Kuroda T, Rabkin SD, Martuza RL. Effective treatment of tumors with strong beta-catenin/T-cell factor activity by transcriptionally targeted oncolytic herpes simplex virus vector. Cancer Res 2006; 66: 10127-10135.

45 Allen C, Vongpunsawad S, Nakamura T, James CD, Schroeder M, Cattaneo R et al. Retargeted oncolytic measles strains entering via the EGFRvIII receptor maintain significant antitumor activity against gliomas with increased tumor specificity. Cancer Res 2006; 66: 11840-11850.

46 Reddy PS, Burroughs KD, Hales LM, Ganesh S, Jones BH, Idamakanti $\mathrm{N}$ et al. Seneca Valley virus, a systemically deliverable oncolytic picornavirus, and the treatment of neuroendocrine cancers. J Natl Cancer Inst 2007; 99: 1623-1633.

47 Wang G, Barrett JW, Stanford M, Werden SJ, Johnston JB, Gao X et al. Infection of human cancer cells with myxoma virus requires Akt activation via interaction with a viral ankyrin-repeat host range factor. Proc Natl Acad Sci USA 2006; 103: 4640-4645.

48 Kurooka M, Kaneda Y. Inactivated Sendai virus particles eradicate tumors by inducing immune responses through blocking regulatory T cells. Cancer Res 2007; 67: 227-236.

49 Raykov Z, Grekova S, Galabov AS, Balboni G, Koch U, Aprahamian $\mathrm{M}$ et al. Combined oncolytic and vaccination activities of parvovirus H-1 in a metastatic tumor model. Oncol Rep 2007; 17: 1493-1499.

50 Freeman AI, Zakay-Rones Z, Gomori JM, Linetsky E, Rasooly L, Greenbaum E et al. Phase I/II trial of intravenous NDV-HUJ oncolytic virus in recurrent glioblastoma multiforme. Mol Ther 2006; 13: 221-228.

51 Laurie SA, Bell JC, Atkins HL, Roach J, Bamat MK, O'Neil JD et al. A phase 1 clinical study of intravenous administration of PV701, an oncolytic virus, using two-step desensitization. Clin Cancer Res 2006; 12: 2555-2562.

52 Kemeny N, Brown K, Covey A, Kim T, Bhargava A, Brody L et al. Phase I, open-label, dose-escalating study of a genetically engineered herpes simplex virus, NV1020, in subjects with metastatic colorectal carcinoma to the liver. Hum Gene Ther 2006; 17: 1214-1224.

$53 \mathrm{Hu}$ JC, Coffin RS, Davis CJ, Graham NJ, Groves N, Guest PJ et al. A phase I study of OncoVEXGM-CSF, a second-generation oncolytic herpes simplex virus expressing granulocyte macrophage colony-stimulating factor. Clin Cancer Res 2006; 12: 6737-6747.

54 Park BH, Hwang TH, Kim SG, Rhee BG, Ahn YJ, Kwon HC et al. A phase I-II clinical trial with JX-594, a targeted and GM-CSFarmed oncolytic poxvirus, by intratumoral injection in patients with liver tumors. AACR-NCI-EORTC International Conference: Molecular Targets and Cancer Therapeutics. American Association for Cancer Research: San Francisco, CA, 2007, pp 116.

55 Thorne SH, Hwang TH, O'Gorman WE, Bartlett DL, Sei S, Kanji $\mathrm{F}$ et al. Rational strain selection and engineering creates a broad-spectrum, systemically effective oncolytic poxvirus, JX-963. J Clin Invest 2007; 117: 3350-3358.

56 Liu TC, Kirn D. Systemic efficacy with oncolytic virus therapeutics: clinical proof-of-concept and future directions. Cancer Res 2007; 67: 429-432.

57 Rots MG, Elferink MG, Gommans WM, Oosterhuis D, Schalk JA, Curiel DT et al. An ex vivo human model system to evaluate specificity of replicating and non-replicating gene therapy agents. J Gene Med 2006; 8: 35-41.

58 Wollmann G, Robek MD, van den Pol AN. Variable deficiencies in the interferon response enhance susceptibility to vesicular stomatitis virus oncolytic actions in glioblastoma cells but not in normal human glial cells. J Virol 2007; 81: 1479-1491.

59 Choi H, Charnsangavej C, Faria SC, Macapinlac HA, Burgess MA, Patel SR et al. Correlation of computed tomography and positron emission tomography in patients with metastatic gastrointestinal stromal tumor treated at a single institution with imatinib mesylate: proposal of new computed tomography response criteria. J Clin Oncol 2007; 25: $1753-1759$. 